\title{
The kinematics of water masers in the stellar molecular outflow source, IRAS 19134+2131
}

\author{
H. Imai ${ }^{1, \star}$, M. Morris ${ }^{2}$, R. Sahai ${ }^{3}$, K. Hachisuka ${ }^{4, \star \star}$, and J. R. Azzollini F. ${ }^{5, \star \star \star}$ \\ 1 Joint Institute for VLBI in Europe, Postbus 2, 7990 AA Dwingeloo, The Netherlands \\ e-mail: imai@jive.nl \\ 2 Department of Physics and Astronomy, University of California, Los Angeles, CA 90095-1562, USA \\ e-mail: morris@astro.ucla.edu \\ 3 Jet Propulsion Laboratory, 4800 Oak Grove Drive, Pasadena, CA 91109, USA \\ e-mail: Raghvendra.Sahai@jpl.nasa.gov \\ ${ }^{4}$ Departamento de Astronomía, Universitat de Valéncia, 46100 Burjassot, Spain \\ e-mail: kazuya.hachisuka@uv.es \\ 5 Instituto de Astrofísica de Canarias, La Laguna, Tenerife 38200, Spain \\ e-mail: jraf@cca.iac.es
}

Received 12 November 2003 / Accepted 4 March 2004

\begin{abstract}
Using the Very Large Array (VLA) and the Very Long Baseline Array (VLBA), we have observed water maser emission in the proto-planetary nebula candidate IRAS 19134+2131, in which the water maser spectrum has two groups of emission features separated in radial velocity by $\sim 100 \mathrm{~km} \mathrm{~s}^{-1}$. The blue-shifted and red-shifted clusters of maser features are clearly separated spatially by $\sim 150$ mas, indicative of a fast collimated flow. However, not all of the maser features are aligned along the axis of the flow, as is seen in the similar high-velocity water maser source, W43A. Comparing the VLA and VLBA maps of the water maser source, we find 4 maser features that were active for 2 years. Using only VLBA data, we identified proper motions for 8 maser features. The full 3D outflow velocity is estimated to be $\sim 130 \mathrm{~km} \mathrm{~s}^{-1}$, indicating that the dynamical age of the flow is only $\sim 50 \mathrm{yr}$. On the basis of the relative positions with respect to the nearby extragalactic reference source, J1925+2106, we also obtain a secular motion of IRAS $19134+2131$ of $\mu_{l}=-4.6 \pm 0.7 \mathrm{mas} \mathrm{yr}^{-1}$ along the Galactic plane toward the Galactic centre. This indicates a "far distance" ( $\geq 16 \mathrm{kpc}$ ) for IRAS $19134+2131$ if the Galactic rotation curve remains flat at $220 \mathrm{~km} \mathrm{~s}^{-1}$.
\end{abstract}

Key words. masers - stars: AGB and post-AGB - stars: distances - stars: kinematics - stars: winds, outflows stars: individual: IRAS 19134+2131

\section{Introduction}

Stellar jets or fast flows have frequently been observed at the late as well as the earliest stages of stellar evolution. In particular, jets during late stellar evolution have been proposed to play an important role in shaping planetary nebulae, considering the frequent bipolarity/multipolarity and point-symmetry observed in proto-planetary and young planetary nebulae $(\mathrm{PNe})$ (e.g., Sahai \& Trauger 1998). The timing and the mechanism of jet formation, however, are still unclear because of the complexity of the final stages of stellar evolution. The circumstellar envelopes of AGB stars have roughly spherical morphologies and

Send offprint requests to: $\mathrm{H}$. Imai,

e-mail: hiroimai@sci.kagoshima-u.ac.jp

* Present address: Department of Physics, Kagoshima University, 1-12-35, Korimoto, Kagoshima 890-0065, Japan.

$\star \star$ Present address: Max-Plank-Institut für Radioastronomie, Auf dem Hügel 69, 53121 Bonn, Germany

$\star \star \star$ Worked in ASTRON/JIVE summer studentship 2003. slow expansion velocities (typically $10-20 \mathrm{~km} \mathrm{~s}^{-1}$ ). However, these envelopes are transformed into proto-planetary nebulae (PPNe) with collimated fast $\left(>100 \mathrm{~km} \mathrm{~s}^{-1}\right)$ outflows and complicated aspherical morphologies during a very short period of their post-AGB evolution ( $\leq 1000 \mathrm{yrs,} \mathrm{e.g.} \mathrm{Lewis} \mathrm{2001).}$

We consider here a member of a small, but exceptionally interesting class of circumstellar water maser sources characterized by extremely large spreads of maser velocities (up to $260 \mathrm{~km} \mathrm{~s}^{-1}$ ) (Likkel et al. 1992). This class of "water fountains" includes only three bona fide objects: IRAS 16342-3814 (hereafter IRAS 1634, Sahai et al. 1999; Morris et al. 2003), W43A (Imai et al. 2002, hereafter Paper I) and IRAS 19134+2131 (hereafter IRAS 1913). This class of sources appears to be closely related morphologically and kinematically to other post-AGB stars revealed by optical, infrared and mm-wave observations (e.g., $\mathrm{OH} 231.8+4.2$ ). Although relatively high velocity OH masers $\left(V_{\exp } \sim 60 \mathrm{~km} \mathrm{~s}^{-1}\right)$ exist in IRAS 1634 (Sahai et al. 1999), the water maser velocities are much higher. For W43A, Paper I described the discovery of a stellar jet traced by 
water masers, which has a highly-collimated morphology and extreme kinematics and which exhibits precession. The water masers seem to delineate the locus of a bipolar precessing jet, whereas the $\mathrm{OH}$ masers are more broadly distributed in systemic latitude. Very likely the $\mathrm{OH}$ masers arise near the surface of an equatorial concentration of gas. Furthermore, the dynamical age of the jet is quite short ( $\geq 40$ yrs at present). Thus, the water fountains probably represent objects in a brief stage between a slow, spherical outflow and a fast axisymmetric outflow. Possibly, the jets in these systems have just been turned on, and they are now punching their ways through the spherical flows, carving out bipolar cavities as they go, and setting the stage for the formation of bipolar planetary nebulae.

Measurements of proper motions as well as radial velocities of water masers provide us the full three-dimensional velocity of a selected portion of the jets. If the source distance is known from other means, this yields the orientation of the jet major axis, which is important for developing a complete picture of the source geometry, including the radial distribution of dust. Unlike most well-studied post-AGB stars whose distances are reasonably well estimated, the distances to the water fountains are relatively unknown. However, if the maser proper motions are measured with respect to extragalactic reference sources, the kinematic distances can be well determined without the ambiguity that occurs when measuring only radial velocities of sources within the Solar circle of the Galaxy.

In this paper, we present the kinematics of water masers associated with IRAS 1913, as revealed by multi-epoch observations with the Very Large Array (VLA) and the Very Long Baseline Array (VLBA). Because the masers are too weak to apply self-calibration $(\leq 1 \mathrm{Jy})$ in the VLBA data and because we need to re-estimate the kinematic distance, we applied the phase-referencing mode to the VLBA observations. Section 2 describes in detail observations and data reduction using NRAO's AIPS software. Section 3 describes the results. Section 4 discusses the implications of the maser kinematics for the evolutionary status and the distances of the water fountains and for the outer Galactic kinematics.

\section{Observations and data reduction}

The VLA observation in the BnA configuration was made at 17:48-18:06 UT on 29 January 2001. The integration duration for IRAS 1913 was about $7 \mathrm{~min}$. Two base-band channels (BBCs) in left circular polarization with a bandwidth of $3.125 \mathrm{MHz}$ covered the blue-shifted and red-shifted maser components. The received signals were correlated and divided into 128 spectral channels in each of the BBCs, corresponding to a velocity spacing of $0.3 \mathrm{~km} \mathrm{~s}^{-1}$ in each of the spectral channels. 3C 295 was observed for the flux density scale and the complex bandpass calibration. The phase calibrator J1931+227 was observed for one minute twice and adopted as a position reference for IRAS 1913.

We did VLA data reduction with the normal procedure, applying self-calibration for the continuum calibrator. The flux density of 3C 295 was assumed to be $0.94 \mathrm{Jy}$ at $22.2 \mathrm{GHz}$. We used a uniformly-weighted synthesized beam of 180 mas $\times 80$ mas with a major axis at a position angle of $-63^{\circ}$ to make image cubes. The detection limit was $0.16 \mathrm{Jy}^{\mathrm{beam}}{ }^{-1}$ at 5- $\sigma$ noise level in a velocity channel without bright maser emission. Two types of image cubes of the IRAS 1913 masers were created; first with self-calibration using the maser data in a spectral channel at $V_{\mathrm{LSR}}=-15.8 \mathrm{~km} \mathrm{~s}^{-1}$ and second with phase-referencing using the data of $\mathrm{J} 1931+227$. The former image cube had better mapping quality because the phase fluctuations due to the atmosphere were well removed. Using the latter image cube, we obtained the absolute coordinates of the maser feature having a velocity of $V_{\mathrm{LSR}}=-15.8 \mathrm{~km} \mathrm{~s}^{-1}, \alpha_{2000}=$ $19^{\mathrm{h}} 15^{\mathrm{m}} 35^{\mathrm{s}} .2162 \pm 0^{\mathrm{s}} .0003, \delta_{2000}=+21^{\circ} 36^{\prime} 33^{\prime \prime} \cdot 916 \pm 0.0^{\prime} 003$.

The VLBA observations were made on 4 January and 7 March 2003 for a total of $10 \mathrm{~h}$ per epoch. NRAO 512 and J2148+0657 were observed for 6 min every 45 min for phase and bandpass calibration. The phase-referencing mode was adopted, in which each antenna nodded between the phasereference and target maser sources in a period of $60 \mathrm{~s}$. The onsource duration in each of the periods was shorter than $20 \mathrm{~s} \mathrm{de}-$ pending on the target-reference separation and antenna zenith angle. J1910+2305 and J1925+2106 (hereafter J1925) were observed as phase-reference sources in different hour angles, but only J1925, 2.5 away from IRAS 1913, was well detected in each of the periods. As a result, the effective coherent integration of the maser data was made for $\sim 2.5 \mathrm{~h}$. The received signals were recorded in 4 BBCs in dual circular polarization with a bandwidth of $4 \mathrm{MHz}$. Two of the BBCs covered the velocities of the red-shifted and blue-shifted maser components. The recorded data were correlated with the Socorro FX correlator in an integration period of $2 \mathrm{~s}$. Each of the BBC data were divided into 256 spectral channels, corresponding to a velocity spacing of $0.2 \mathrm{~km} \mathrm{~s}^{-1}$ in each of the spectral channels. The following coordinates of IRAS 1913 were adopted in the data correlation: $\alpha_{2000}=19^{\mathrm{h}} 15^{\mathrm{m}} 35^{\mathrm{s}} \cdot 2150, \delta_{2000}=+21^{\circ} 36^{\prime} 33^{\prime \prime} \cdot 900$.

In VLBA data reduction, we applied the procedures for the phase-referencing mode (e.g. Beasley \& Conway 1995; Reid et al. 1999). First, residual delay/delay-rate solutions were obtained from fringe fitting for scans of NRAO 512 and $\mathrm{J} 2148+0657$. In this stage, we estimated that the residual delay solutions had uncertainties less than $3 \mathrm{nsec}$. Then fringe fitting was performed for scans of J1925. Most of the residual delayrate solutions were smaller than $10 \mathrm{mHz}$; we could thus avoid $2 \pi$-n-radian ambiguity of fringe-phase interpolation between the successive calibrator scans. All of the calibration solutions obtained for J1925, including solutions in the self-calibration, were applied to the maser data. We used a naturally-weighted synthesized beam of 0.98 mas $\times 0.37$ mas with a major axis at a position angle of $-13^{\circ}$ to make image cubes. The detection limit was $60 \mathrm{mJy}^{-1}$ beam $^{-1}$ at 5- $\sigma$ noise level in a velocity channel without bright maser emission.

The maser astrometry is affected by the brightness structure of J1925 and by relative difference in the excess path delays in the atmosphere between the reference and the maser sources due to different zenith angles. These are described in Appendices A and B. J1910+2305 was also marginally detected after applying fringe-fitting and self-calibration solutions for the maser data, but which is not described in detail here. 


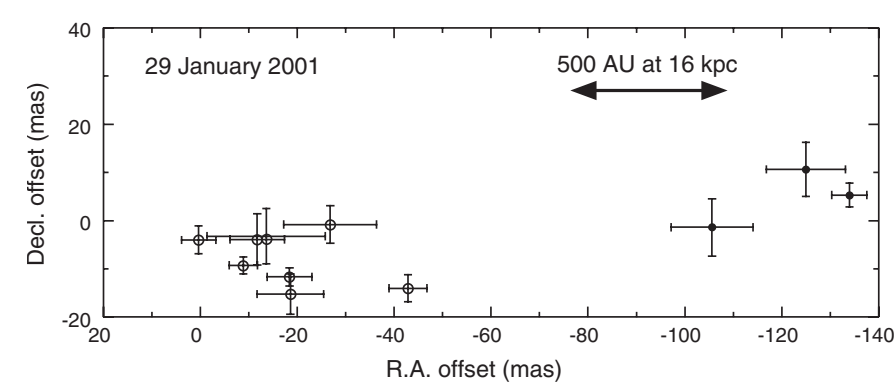

Fig. 1. Distribution of water maser features in IRAS 19134+2131 observed with the VLA. An uncertainty of a maser feature position is indicated by the size of a cross. The blue-shifted $\left(-122 \mathrm{~km} \mathrm{~s}^{-1} \leq\right.$ $\left.V_{\mathrm{LSR}} \leq-117 \mathrm{~km} \mathrm{~s}^{-1}\right)$ and red-shifted $\left(-37 \mathrm{~km} \mathrm{~s}^{-1} \leq V_{\mathrm{LSR}} \leq\right.$ $-10 \mathrm{~km} \mathrm{~s}^{-1}$ ) maser features with respect to the source systemic velocity, $V_{\mathrm{LSR}}=-67 \mathrm{~km} \mathrm{~s}^{-1}$, are shown in filled and opened circles, respectively.
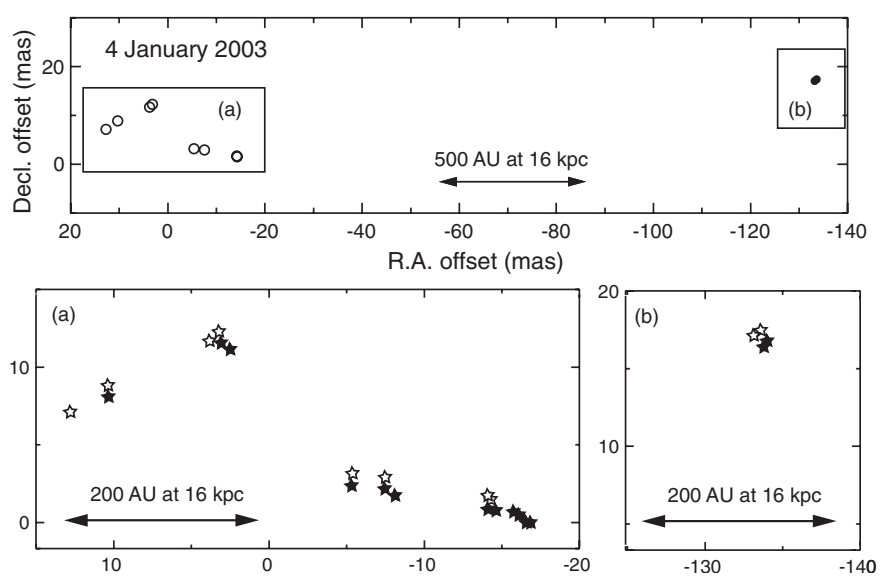

Fig. 2. Distributions of water maser features in IRAS 19134+2131 observed with the VLBA. Upper: radial velocity distribution of the maser features on 4 January 2003. The blue-shifted $\left(-122 \mathrm{~km} \mathrm{~s}^{-1} \leq\right.$ $\left.V_{\mathrm{LSR}} \leq-117 \mathrm{~km} \mathrm{~s}^{-1}\right)$ and red-shifted $\left(-37 \mathrm{~km} \mathrm{~s}^{-1} \leq V_{\mathrm{LSR}} \leq\right.$ $-10 \mathrm{~km} \mathrm{~s}^{-1}$ ) maser features with respect to the source systemic velocity, $V_{\mathrm{LSR}}=-67 \mathrm{~km} \mathrm{~s}^{-1}$, are shown in filled and opened circles, respectively. Lower: sub panels zooming up the blue-shifted a) and redshifted b) clusters of maser features. Opened and filled stars indicate the feature locations on 4 January and 7 March in 2003, respectively.

\section{Results}

\subsection{The IRAS 19134+2131 flow traced by water masers}

Figures 1 and 2 show the distributions of the water maser features in IRAS 1913 observed with the VLA and the VLBA, respectively. Tables 1 and 2 give parameters of maser features detected with the VLA and the VLBA, respectively. The detection limits of the VLA and the VLBA observations were roughly equal to each other, and the maser distributions were roughly similar among the observations. Because of the large VLA synthesized beam and insufficient accuracy of maser feature positions, it is difficult to exactly trace the same maser features in the VLA and VLBA maps unless a maser feature is spatially isolated. Nevertheless, we found at least 4 maser
Table 1. Parameters of water maser features detected in the VLA observation.

\begin{tabular}{rrccc}
\hline \hline $\begin{array}{r}V_{\text {LSR }} \\
\left(\mathrm{km} \mathrm{s}^{-1}\right)\end{array}$ & $\begin{array}{c}\Delta \alpha \\
(\mathrm{mas})\end{array}$ & $\begin{array}{c}\Delta \delta \\
(\mathrm{mas})\end{array}$ & $\begin{array}{c}I_{\text {Peak }} \\
\left(\mathrm{Jy} \mathrm{beam}^{-1}\right)\end{array}$ & $\begin{array}{c}{ }^{a} \Delta V \\
\left(\mathrm{~km} \mathrm{~s}^{-1}\right)\end{array}$ \\
\hline-118.9 & $-134 \pm 4$ & $5 \pm 2$ & 1.38 & 2.3 \\
-115.3 & $-106 \pm 8$ & $-1 \pm 6$ & 0.22 & 0.2 \\
-114.9 & $-125 \pm 8$ & $11 \pm 6$ & 0.34 & 0.7 \\
-36.6 & $-43 \pm 4$ & $-14 \pm 3$ & 0.78 & 1.0 \\
-21.5 & $-19 \pm 7$ & $-15 \pm 4$ & 0.41 & 1.7 \\
-18.7 & $-27 \pm 10$ & $-1 \pm 4$ & 0.32 & 0.7 \\
-17.0 & $-12 \pm 6$ & $-4 \pm 5$ & 1.16 & 1.3 \\
$b_{-15.9}$ & $0 \pm 4$ & $-4 \pm 3$ & 1.77 & 1.7 \\
-14.1 & $-14 \pm 12$ & $-3 \pm 6$ & 0.33 & 1.0 \\
-13.2 & $-9 \pm 3$ & $-9 \pm 2$ & 1.11 & 1.0 \\
-12.5 & $-18 \pm 5$ & $-12 \pm 2$ & 0.77 & 1.0 \\
\hline
\end{tabular}

${ }^{a}$ Full velocity coverage of the maser feature.

${ }^{b}$ Maser feature containing the maser spot (located at the map origin) in the phase-reference velocity channel at $V_{\mathrm{LSR}}=-15.8 \mathrm{~km} \mathrm{~s}^{-1}$.

features that had been active for 2 years. The existence of maser features having lifetimes longer that one year is supported by the single-dish monitoring observations by Likkel et al. (1992), and helpful for high-precision measurements of maser proper motions.

The blue-shifted and red-shifted clusters of maser features are spatially well distinguished, indicating the existence of a collimated flow. The projected flow length is $\sim 150$ mas, corresponding to $\sim 2400 \mathrm{AU}$ at a distance of $16 \mathrm{kpc}$ (see Sect. 4.2). Note that the VLA and VLBA observations did not reveal any straight line morphology in the maser feature clusters such as that seen in the W43A jet; the IRAS 1913 flow is thus less collimated than the W43A jet (Paper I). This is not a result of sensitivity bias for the detected maser features.

Using only VLBA data, we identified 8 proper motions of the water maser features (see Fig. 2 and Table 2). Note that, as described in Appendix A, the variation in the J1925 brightness structure introduces a coordinate drift of less than $15 \mu$ as. We measured at first mean proper motions of the blue-shifted and red-shifted clusters, then estimated a mean proper motion of the clusters as a secular motion that is discussed in the next section. An expansion motion of the flow is obtained as a relative motion of one of the two clusters with respect to the secular motion. Thus, we obtain an expansion rate of $1.5 \pm 0.5 \mathrm{mas} \mathrm{yr}^{-1}$ in one direction in a position angle of about $74^{\circ}$ east from north (weighted by the inverses of proper motion errors), corresponding to $\sim 120 \mathrm{~km} \mathrm{~s}^{-1}$ on the sky plane. An uncertainty of the value was estimated from the standard deviation of the proper motions in two of the maser clusters. Combined with an expansion velocity of $\sim 50 \mathrm{~km} \mathrm{~s}^{-1}$ along the line-of-sight, we obtain a 3D flow speed of $\sim 130 \mathrm{~km} \mathrm{~s}^{-1}$, an inclination of $\sim 25^{\circ}$ with respect to the sky plane, and a dynamical age of the flow of $\sim 50$ yrs assuming constant proper motions with time. 
Table 2. Parameters of water maser features detected in the VLBA observations.

\begin{tabular}{|c|c|c|c|c|c|c|c|c|c|c|c|}
\hline \multicolumn{5}{|c|}{4 January 2003} & \multicolumn{5}{|c|}{7 March 2003} & \multicolumn{2}{|c|}{${ }^{b}$ Proper motion } \\
\hline $\begin{array}{r}V_{\mathrm{LSR}} \\
\left(\mathrm{km} \mathrm{s}^{-1}\right) \\
\end{array}$ & $\begin{array}{c}\Delta \alpha \\
\text { (mas) }\end{array}$ & $\begin{array}{c}\Delta \delta \\
\text { (mas) }\end{array}$ & $\begin{array}{c}I_{\text {Peak }} \\
\left(\mathrm{Jy} \mathrm{beam}^{-1}\right)\end{array}$ & $\begin{array}{c}{ }^{a} \Delta V \\
\left(\mathrm{~km} \mathrm{~s}^{-1}\right)\end{array}$ & $\begin{array}{r}V_{\mathrm{LSR}} \\
\left(\mathrm{km} \mathrm{s}^{-1}\right)\end{array}$ & $\begin{array}{c}\Delta \alpha \\
\text { (mas) }\end{array}$ & $\begin{array}{c}\Delta \delta \\
\text { (mas) }\end{array}$ & $\begin{array}{c}I_{\text {Peak }} \\
\left(\mathrm{Jy} \mathrm{beam}^{-1}\right)\end{array}$ & $\begin{array}{c}{ }^{a} \Delta V \\
\left(\mathrm{~km} \mathrm{~s}^{-1}\right)\end{array}$ & $\begin{array}{c}\mu_{\alpha} \\
\left(\operatorname{mas~yr}^{-1}\right)\end{array}$ & $\begin{array}{c}\mu_{\delta} \\
\left(\operatorname{mas~yr}^{-1}\right)\end{array}$ \\
\hline \multicolumn{3}{|c|}{$-120.4-133.54 \pm 0.0217 .41 \pm 0.04$} & 0.32 & 1.9 & \multicolumn{3}{|c|}{$-120.5-134.01 \pm 0.0616 .83 \pm 0.15$} & 0.05 & 0.6 & \multicolumn{2}{|c|}{$-2.82 \pm 0.06-3.40 \pm 0.16$} \\
\hline \multicolumn{3}{|c|}{$-118.1-133.19 \pm 0.0517 .06 \pm 0.08$} & 0.22 & 1.7 & \multicolumn{3}{|c|}{$-118.1-133.72 \pm 0.0316 .34 \pm 0.05$} & 0.11 & 1.1 & \multicolumn{2}{|c|}{$-3.18 \pm 0.06-4.32 \pm 0.09$} \\
\hline-22.2 & \multicolumn{2}{|c|}{$3.22 \pm 0.0712 .30 \pm 0.07$} & 0.49 & 1.9 & -22.1 & \multicolumn{2}{|c|}{$3.13 \pm 0.0411 .52 \pm 0.05$} & 0.31 & 1.5 & \multicolumn{2}{|c|}{$-0.54 \pm 0.07-4.68 \pm 0.09$} \\
\hline$\ldots$ & $\ldots$ & $\ldots$ & $\ldots$ & $\ldots$ & -22.2 & $2.55 \pm 0.03$ & $11.09 \pm 0.04$ & 0.12 & 1.1 & \multirow{2}{*}{$\ldots$} & $\ldots$ \\
\hline-20.6 & $3.85 \pm 0.08$ & $11.68 \pm 0.18$ & 0.08 & 0.8 & $\ldots$ & $\ldots$ & $\ldots$ & $\ldots$ & $\ldots$ & & $\ldots$ \\
\hline-18.9 & $-14.24 \pm 0.07$ & $1.50 \pm 0.04$ & 0.13 & 1.1 & -19.1 & $-14.11 \pm 0.04$ & $0.76 \pm 0.05$ & 0.10 & 0.6 & \multicolumn{2}{|c|}{$0.78 \pm 0.08-4.48 \pm 0.06$} \\
\hline$\ldots$ & $\ldots$ & $\ldots$ & $\ldots$ & $\ldots$ & -18.0 & $-15.94 \pm 0.06$ & $0.39 \pm 0.03$ & 0.31 & 1.7 & $\ldots$ & $\ldots$ \\
\hline & $\ldots$ & $\ldots$ & $\ldots$ & $\ldots$ & -17.9 & $-16.51 \pm 0.02$ & $-0.05 \pm 0.04$ & 0.13 & 0.8 & $\ldots$ & $\ldots$ \\
\hline-17.0 & $12.82 \pm 0.05$ & $7.14 \pm 0.07$ & 0.11 & 0.6 & $\ldots$ & $\ldots$ & $\ldots$ & $\ldots$ & $\ldots$ & $\ldots$ & $\ldots$ \\
\hline$\ldots$ & $\ldots$ & $\ldots$ & $\ldots$ & $\ldots$ & -16.9 & $-16.73 \pm 0.05$ & $-0.03 \pm 0.05$ & 0.13 & 0.8 & $\ldots$ & $\ldots$ \\
\hline$\ldots$ & $\ldots$ & $\ldots$ & $\ldots$ & $\ldots$ & -16.7 & $-16.15 \pm 0.02$ & $0.37 \pm 0.02$ & 0.28 & 0.8 & $\ldots$ & $\ldots$ \\
\hline$\ldots$ & $\ldots$ & $\ldots$ & $\ldots$ & $\ldots$ & -16.0 & $-16.26 \pm 0.02$ & $0.36 \pm 0.04$ & 0.28 & 1.1 & $\ldots$ & $\ldots$ \\
\hline$\ldots$ & $\ldots$ & $\ldots$ & $\ldots$ & $\ldots$ & -15.9 & $-16.85 \pm 0.07$ & $-0.04 \pm 0.05$ & 0.11 & 1.1 & $\ldots$ & $\ldots$ \\
\hline$\ldots$ & $\ldots$ & $\ldots$ & $\ldots$ & $\ldots$ & -15.8 & $-15.86 \pm 0.12$ & $0.57 \pm 0.10$ & 0.11 & 1.7 & $\ldots$ & $\ldots$ \\
\hline$\ldots$ & $\ldots$ & $\ldots$ & $\ldots$ & $\ldots$ & -15.4 & $-14.63 \pm 0.05$ & $0.82 \pm 0.05$ & 0.12 & 0.6 & $\ldots$ & $\ldots$ \\
\hline-14.8 & $10.40 \pm 0.03$ & $8.84 \pm 0.02$ & 0.33 & 0.8 & -14.8 & $10.38 \pm 0.02$ & $8.04 \pm 0.03$ & 0.25 & 0.8 & $-0.12 \pm 0.04$ & $.40 \pm 0.04$ \\
\hline-14.0 & $-14.15 \pm 0.01$ & $1.66 \pm 0.02$ & 1.19 & 1.5 & -13.9 & $-14.11 \pm 0.02$ & $0.89 \pm 0.04$ & 0.52 & 1.5 & $0.24 \pm 0.02$ & $.62 \pm 0.04$ \\
\hline-12.2 & $-5.34 \pm 0.02$ & $3.11 \pm 0.03$ & 0.19 & 0.8 & -12.0 & $-5.27 \pm 0.01$ & $2.33 \pm 0.04$ & 0.20 & 0.8 & $0.42 \pm 0.02$ & $.68 \pm 0.05$ \\
\hline-11.1 & $-7.50 \pm 0.01$ & $2.87 \pm 0.01$ & 1.79 & 2.1 & -11.1 & $-7.51 \pm 0.01$ & $2.10 \pm 0.01$ & 2.01 & 1.9 & $-0.06 \pm 0.01$ & $.62 \pm 0.01$ \\
\hline$\ldots$ & $\ldots$ & $\ldots$ & $\ldots$ & $\ldots$ & -11.1 & $-8.08 \pm 0.01$ & $1.69 \pm 0.02$ & 0.76 & 1.3 & $\ldots$ & $\ldots$ \\
\hline
\end{tabular}

${ }^{a}$ Full velocity coverage of the maser feature.

${ }^{b}$ Errors contributing from thermal noise and feature structure are taken into account.

\subsection{Galactic rotation at the IRAS 19134+2131 location}

Adopting a mean motion between the blue-shifted and redshifted clusters of maser features as the secular proper motion of the IRAS 1913 water maser source, we obtained this secular motion to be (weighted by inverses of proper motion errors),

$\mu_{\alpha}=-1.5 \pm 0.2( \pm 0.2)$ mas $\mathrm{yr}^{-1}$,

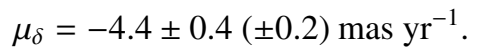

The error in the parentheses indicates an expected systematic error. These equatorial velocity components are converted to Galactic coordinates as follows,

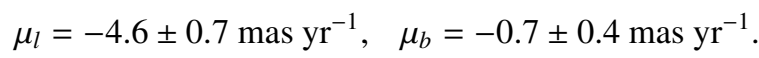

Thus, the secular motion is roughly along the Galactic plane toward the Galactic center, or dominated by Galactic rotation ${ }^{1}$. Since the radial velocity of IRAS 1913 is negative, we unambiguously conclude that it lies at a "far distance" rather than a "near distance". The far distance of IRAS 1913 is also consistent with its secular motion, which is smaller than that of

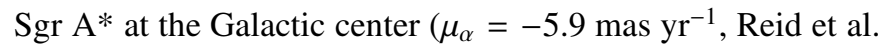
1999).

\section{Discussion}

\subsection{What are the water fountains?}

As described in Sect. 3.1, the IRAS 1913 flow, has similar physical parameters as those for another "water fountain",

\footnotetext{
${ }^{1}$ A possible effect of the annual parallax ( $<0.1$ mas) is smaller than the proper motion uncertainty.
}

the W43A jet, which has a projected length of $\sim 1700$ AU with an inclination of $\simeq 39^{\circ}$, and a dynamical age of $\sim 35$ yrs in the year $1994^{2}$ (Paper I). The IRAS 1634 flow is also similar: its projected length of the flow is $\sim 4500 \mathrm{AU}$ and the dynamical age is $\leq 100$ yrs (Morris et al. 2003). Such a short time scale $(\leq 100 \mathrm{yrs})$ of the three water fountains is consistent with their rarity; only these sources have velocity separations over $100 \mathrm{~km} \mathrm{~s}^{-1}$ (Likkel et al. 1992, see also the latest Arcetri cata$\log$ of water maser sources by Valdettaro et al. 2002).

These characteristics imply that highly collimated jets of molecular gas traced by water maser emission are seen only during such a short period. Moreover, strong collimation of a stellar jet might occur during much shorter time scale; only the W43A jet with the shortest dynamical age exhibits a strong collimation. The true morphology of the IRAS 1913 outflow, however, must be confirmed by detecting and following more maser features in order to examine these hypothesis. After all, a collimated jet of molecular gas traced by water maser emission is likely to quickly disappear after the above-mentioned time scale. We speculate that the jet has moved far enough out through the ambient, lower-velocity outflows so that the gas density is too low to excite maser activity in the high velocity jet by shocks (cf. Elitzur et al. 1989, 1992). This speculation is consistent with the observation of the PPN K3-35 (Miranda et al. 2001) in which a collimated jet traced by cm-wave radio continuum emission has a relatively long dynamical age of

${ }^{2}$ In Paper I, the dynamical age of the W43A jet was incorrectly calculated, the projected length of the jet divided by the 3D jet speed. Here, the dynamical age is recalculated, the projected length divided by the projected jet speed. 
$\sim 800$ yrs, and only low-velocity water maser components are found.

There is a close alignment of the bipolarity axis in the optical image to the axis of the water maser jet in both IRAS 1634 (Morris et al. 2003) and IRAS 1913 (Sahai in private communication). This alignment strongly supports the idea that the operation of these jets has created the bipolar morphology in these objects. Thus, the water fountain nebulae are important objects for investigating the mechanisms involved in shaping planetary nebulae at their earliest stages. High spatial-resolution (interferometric) observations of $\mathrm{CO}$ emission from these objects, can further strengthen this hypothesis by enabling us to estimate the mass-loss rates and speeds of the molecular outflows and their correlations with the properties of the water fountain jets.

\subsection{Implications for the source distance and Galactic-rotation velocity}

The radial and transverse velocities of the source observed, $V_{\mathrm{r}}$ and $V_{\mathrm{t}}$ respectively, are expressed as follows,

$$
\begin{aligned}
V_{\mathrm{r}} & =\left(\frac{R_{\odot}}{R} V^{\mathrm{rot}}-V_{\odot}^{\mathrm{rot}}\right) \sin l \cos b+V_{\mathrm{r}}^{\mathrm{pec}}, \\
V_{\mathrm{t}} & =a \mu_{l} r \\
& =-\left[V^{\mathrm{rot}} \frac{r}{R}+\left(V_{\odot}^{\mathrm{rot}}-\frac{R_{\odot}}{R} V^{\mathrm{rot}}\right) \cos l\right] \cos b+V_{\mathrm{t}, \|}^{\mathrm{pec}},
\end{aligned}
$$

where $r$ is the heliocentric distance of the maser source in units of kiloparsecs, $R$ and $R_{\odot}$ are the Galactocentric distances of the source and the Sun, $V^{\text {rot }}$ and $V_{\odot}^{\text {rot }}$ are the full Galactic rotation velocities at their locations, respectively, $a=$ $4.74 \mathrm{~km} \mathrm{~s}^{-1} \mathrm{yr} \mathrm{kpc}^{-1}$ is the conversion factor from the measured proper motion to the transverse velocity, $\mu_{l}$ is the proper motion

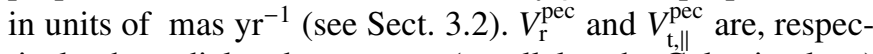
tively, the radial and transverse (parallel to the Galactic plane) components of a peculiar motion of the source, which are unknown and expressed as a deviation from circular Galactic rotation. The variables $R$ and $r$ are related:

$R^{2}=r^{2}+R_{\odot}^{2}-2 r R_{\odot} \cos l, \quad R>R_{\odot}$.

From these equations, $V^{\text {rot }}$ can be expressed in two forms as functions of $R$ or $r$ as follows,

$$
\begin{aligned}
V^{\mathrm{rot}}= & \frac{R}{R_{\odot}}\left(\frac{V_{\mathrm{r}}-V_{\mathrm{r}}^{\mathrm{pec}}}{\sin l \cos b}+V_{\odot}^{\mathrm{rot}}\right), \\
V^{\mathrm{rot}}= & -\left(a \mu_{l} R_{\odot}+V_{\odot}^{\mathrm{rot}} \cos b\right) \frac{R \cos l}{y \cos b} \\
& -\frac{a \mu_{l} R}{\cos b}+\frac{R V_{\mathrm{t}}^{\mathrm{pec}, \|}}{y \cos b},
\end{aligned}
$$

where $y=\left(R^{2}-R_{\odot}^{2} \sin ^{2} l\right)^{1 / 2}$.

If the peculiar velocity components are known, then the solution for $R$ and $V^{\text {rot }}$ can be found by observing both a radial velocity and a proper motion for the maser source, and by assuming a Galactocentric distance to the Sun, $R_{\odot}=8 \mathrm{kpc}$, and a Galactic rotation velocity at the Sun, $V_{\odot}^{\mathrm{rot}}=220 \mathrm{~km} \mathrm{~s}^{-1}$.

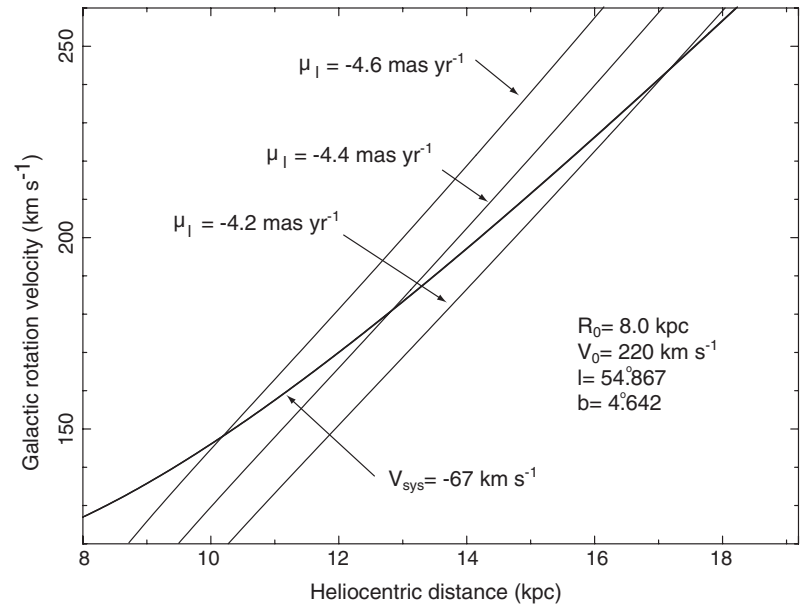

Fig. 3. Possible heliocentric distance, $r$, and Galactic rotation velocity, $V^{\text {rot }}$, of IRAS $19134+2131$ located at $(l, b)=\left(54^{\circ} .867,4.642\right)$. Three slopes of the function corresponding to the proper motions, $\mu_{l}=-4.6$,

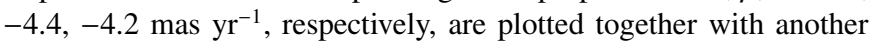
curve corresponding to the source radial velocity $V_{\mathrm{LSR}}=-67 \mathrm{~km} \mathrm{~s}^{-1}$.

Figure 3 suggests that an accuracy of the proper motion bet-

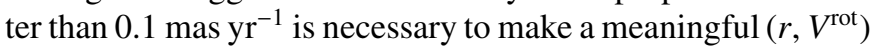
measurement. This accuracy is possible with current very long baseline interferometry (VLBI) techniques and with a longer time baseline of the monitoring observations. The source proper motion, $\mu_{l}=-4.6 \pm 0.7$ mas $\mathrm{yr}^{-1}$, gives a Galactic rotation velocity at the source location, $V^{\text {rot }} \sim 150 \mathrm{~km} \mathrm{~s}^{-1}$ and a heliocentric distance of $10 \mathrm{kpc}$. Taking into account a possible flat Galactic rotation curve $\left(V^{\mathrm{rot}}=220 \mathrm{~km} \mathrm{~s}^{-1}\right)$, however,

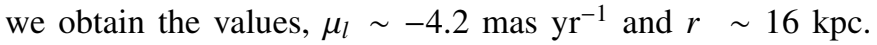
To meaningfully perform the $\left(r, V^{\text {rot }}\right)$ estimation, a $\mu_{l}$-accuracy

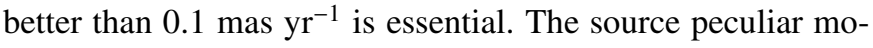
tion is also an unknown. In the Solar neighborhood, AGB stars have dispersions in the peculiar motions of $\sigma_{r} \simeq 22 \mathrm{~km} \mathrm{~s}^{-1}$ and $\sigma_{t} \simeq 25 \mathrm{~km} \mathrm{~s}^{-1}$ in the radial and transverse directions, respectively (Mihalas \& Binney 1981). These dispersions cause uncertainties of up to $6 \mathrm{kpc}$ and $60 \mathrm{~km} \mathrm{~s}^{-1}$ in a heliocentric distance and a Galactic rotation, respectively. At the outer Galaxy, a peculiar motion due to the Galactic warp is also expected (the warping disk traced by $\mathrm{H}$ I emission was recently well analyzed by Nakanishi \& Sofue 2003).

Nevertheless, the analyses described above are still useful to elucidate the outer Galactic rotation curve and the source location in the Galaxy. Future statistical analyses may reveal a mean Galactic rotation curve based on the kinematics of a group of AGB/post-AGB stars and stellar peculiar motions can be determined as deviations from the mean Galactic rotation.

\section{Summary}

(6) The present VLA and VLBA observations have revealed the distribution and the kinematics of the water masers in IRAS 1913 and the secular motion of IRAS 1913 following the Galactic rotation. The existence of a well-collimated flow is supported by the blue-shifted and red-shifted maser components that are well spatially separated. The measured flow

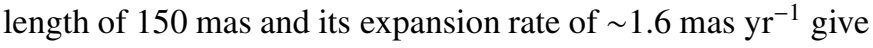


a flow dynamical age of $\sim 50$ yrs. Compared with the jet parameters of other "water fountains", W43A and IRAS 1634, and rarity of this kind of objects, we speculate that the water fountains are seen during a very short period ( $\leq 100 \mathrm{yrs})$. It is most likely that, after this time, the aligned high-velocity water maser features disappear and only the low-velocity components of water maser emission exist, until eventual photoionization of the circumstellar envelope. The secular motion of IRAS 1913 along the Galactic plane, $\mu_{l} \sim-4.6$ mas $\mathrm{yr}^{-1}$, puts IRAS 1913 at a "far distance" $(\sim 10 \mathrm{kpc}$ depending on the Galactic rotation curve). More accurate measurements of the IRAS 1913 secular motion and those of similar, distant objects will enable us to estimate not only the distance to these objects but also the outer Galactic rotation curve and interesting characteristics in their peculiar motions. Long-term activity of individual maser features over 2 yrs should support such measurements.

Acknowledgements. Authors acknowledge Dr. Vivek Dhawan for helping our VLBA observations as a technical contact person. They also thank Dr. Huib Jan van Langevelde for fruitful suggestions relating to the data reduction. The NRAO is a facility of the National Science Foundation of the USA, operated under a cooperative agreement by Associated Universities, Inc.

\section{Appendix A: The brightness structure of the $\mathrm{J} 1925+2106$}

The celestial coordinates for the IRAS 1913 water masers in this paper are well fixed by the extragalactic reference frame using the reference source J1925. Figure A.1 shows the brightness structure of J1925 at the two epochs - a bright core, very close to the map origin (component $A$ ), and one or two more fainter components, are visible. The second brightest component (component $B$ ) is located at a position angle of about $-100^{\circ}$ from north with respect to component $A$, which is consistent with the direction of the elongated distribution of radio emission components seen in longer wavelength bands (e.g., Fomalont et al. 2000). Because the self-calibration process puts the coordinate origin at the mean center of the J1925 brightness structure, the stability of the coordinates depends on the time variation of the J1925 brightness structure.

Table A.1 gives the locations of components $A$ and $B$, which were estimated by Gaussian model fitting using the AIPS task JMFIT. Component $A$ is offset by $\sim 40 \mu$ as from the map origin, but has been stable within $\sim 6 \mu$ as between the two epochs. This component, likely associated with a massive black hole, is suitable as a position reference for the astrometry of the IRAS 1913 masers. On the other hand, component $B$ may exhibit a proper motion of $108 \pm 140 \mu$ as. This motion will be better estimated by additional observations covering a longer time interval $(\leq 1 \mathrm{yr})$. The third brightest component was marginally seen to the west of the brightest component,

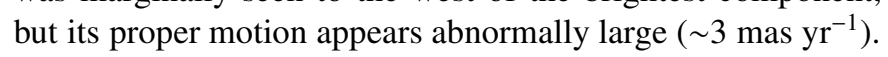
We judge that the significance of this result is still low.

The coordinates are also affected by the parameters adopted in data reduction. We performed the model fitting by adopting models having two elliptical Gaussian features for components $A$ and $B$. We prepared three maps with different pixel

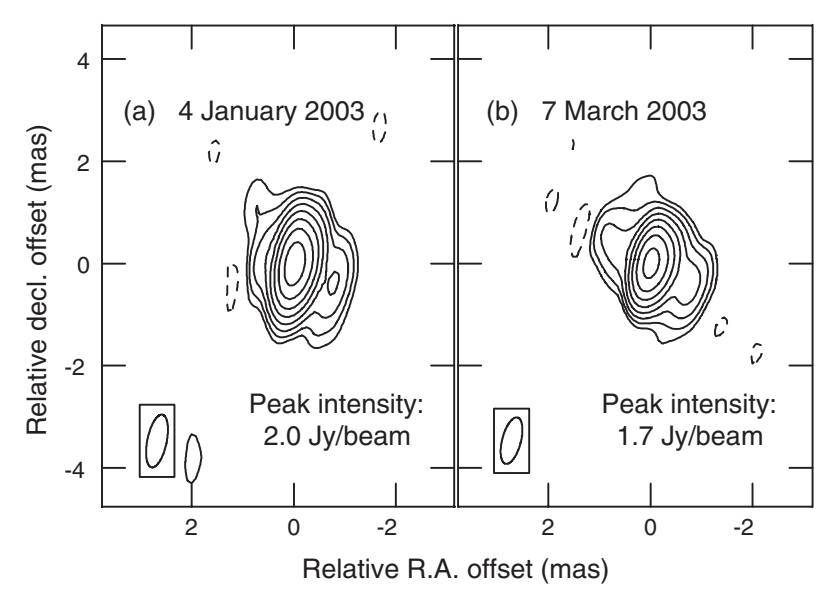

Fig. A.1. Brightness distribution of J1925+2106 at the first a) and second b) epochs. The contour levels are set at $-10,10,20,40,80,160$, 320,640 and $1280 \mathrm{mJy} \mathrm{beam}^{-1}$. A box in the left-bottom corner indicates the synthesized beam.

Table A.1. Locations of the two brightness components in J1925+2106.

\begin{tabular}{lcccc}
\hline \hline & \multicolumn{2}{c}{ Relative offsets } & \multicolumn{2}{c}{ Difference between } \\
& on 4 Jan. 2003 & \multicolumn{2}{c}{ 7 Mar. and 4 Jan. } \\
\hline & $\Delta \alpha$ & $\Delta \delta$ & $\Delta \alpha$ & $\Delta \delta$ \\
Comp. & $(\mu$ as $)$ & $(\mu$ as $)$ & $(\mu$ as $)$ & $(\mu$ as $)$ \\
\hline A $\ldots .$. & $-28 \pm 7$ & $-22 \pm 6$ & $5 \pm 10$ & $12 \pm 7$ \\
B $\ldots .$. & $-454 \pm 89$ & $-12 \pm 101$ & $-31 \pm 104$ & $-94 \pm 119$ \\
\hline
\end{tabular}

sizes and performed the fitting many times by changing fit field sizes and a-priori locations of the two components. The standard deviations of the estimated component locations should correspond to uncertainties in the locations, which are given in Table A.1. Thus we conclude that the assumption of coordinate stability is uncertain by $\leq 15 \mu$ as because of brightness frame variation.

\section{Appendix B: Astrometric error due to different excess path delays between two sources}

In contrast to relative proper motions among maser features, proper motions with respect to a reference source, a few degrees away from the maser source, have worse accuracies because of additional errors in measured relative phase delays due to the unknown difference of atmospheric excess path delays between the two sources. For the VLBA data, this additional phase-delay error, $\Delta \tau$, was evaluated by Reid et al. (1999) using the following equation,

$\Delta \tau \approx \tau_{0} \sec Z \tan Z \Delta Z$

where $\tau_{0} \sim 0.1 \mathrm{~ns}$ is the phase-delay error at the zenith yielded in the VLBA correlation model, $Z$ the zenith angle of the one of the two observed sources, $\Delta Z$ the zenith angle difference between the two observed sources. In the present paper adopting $Z \sim 30^{\circ}, \Delta Z \sim 2.5^{\circ}$, a typical phase-delay error of about $2.2 \times 10^{-12} \mathrm{~s}$ is obtained, which corresponds to a position error 
of $130 \mu$ as when observing with a $1000-\mathrm{km}$ baseline. Thus a systematic error of the order of $100 \mu$ as is expected.

\section{References}

Beasley, J. A., \& Conway, J. E. 1995, Very Long Baseline Interferometry and the VLBA, ed. J. A. Zensus, P. J. Diamond, \& P. J. Napier (San Francisco: ASP), ASP Conf. Ser., 82, 328

Fomalont, E. B., Frey, S., Paragi, Z., et al. 2000, ApJS, 131, 95

Elitzur, M., Hollenbach, D. J., \& McKee, C. F. 1992, ApJ, 394, 221

Elitzur, M., Hollenbach, D. J., \& McKee, C. F. 1989, ApJ, 346, 983

Imai, H., Obara, K., Diamond, P. J., Omodaka, T., \& Sasao, T. 2002, Nature, 417, 829

Lewis, B. M. 2001, A\&A, 560, 400
Likkel, L., Morris, M., \& Maddalena, R. J. 1992, A\&A, 256, 581

Mihalas, D., \& Binney, J. 1981, Galactic Astronomy, second edition, Structure and Kinematics (San Francisco: W. H. Freeman and Company), Chap. 7

Miranda, L. F., Gómez, Y., Anglada, G., \& Torrelles, J. M. 2001, Nature, 414, 284

Morris, M. R., Sahai, R., \& Claussen, M. 2003, RMxAC, 15, 20

Nakanishi, H., \& Sofue, Y. 2003, PASJ, 55, 191

Reid, M. J., Readhead, A. C. S., Vermeulen, R. C., \& Treuhaft, R. N. 1999, ApJ, 524, 816

Sahai, R., Te Lintel Hellert, P., Morris, M., Zijlstra, A., \& Likkel, L. 1999, ApJ, 514, L115

Sahai, R., \& Trauger, J. T. 1998, AJ, 116, 1357

Valdettaro, R., Palla, F., Brand, J., et al. 2002, A\&A, 368, 845 